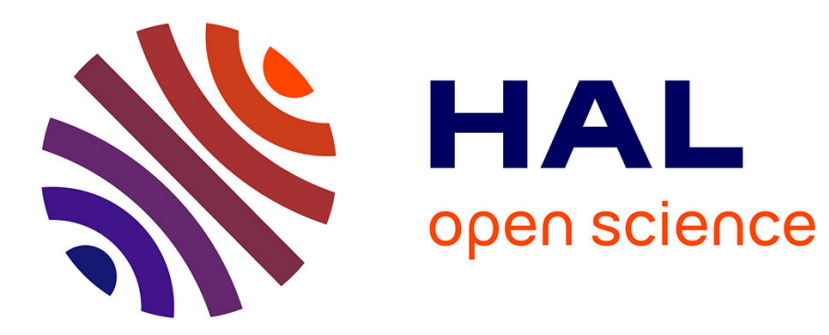

\title{
Les mobilités quotidiennes, un révélateur des inégalités sociospatiales à Ouagadougou
}

Florence Boyer, Vincent Gouëset, Daniel Delaunay

\section{To cite this version:}

Florence Boyer, Vincent Gouëset, Daniel Delaunay. Les mobilités quotidiennes, un révélateur des inégalités sociospatiales à Ouagadougou. Autrepart - Revue de sciences sociales au Sud, 2016. halshs01655739

\section{HAL Id: halshs-01655739 \\ https://shs.hal.science/halshs-01655739}

Submitted on 5 Dec 2017

HAL is a multi-disciplinary open access archive for the deposit and dissemination of scientific research documents, whether they are published or not. The documents may come from teaching and research institutions in France or abroad, or from public or private research centers.
L'archive ouverte pluridisciplinaire HAL, est destinée au dépôt et à la diffusion de documents scientifiques de niveau recherche, publiés ou non, émanant des établissements d'enseignement et de recherche français ou étrangers, des laboratoires publics ou privés. 


\title{
Les mobilités quotidiennes, un révélateur des inégalités sociospatiales à Ouagadougou
}

\author{
Florence Boyer*, Vincent Gouëset**, Daniel Delaunay***
}

La mobilité de tous les jours apparaît dans les sociétés contemporaines comme une valeur et une préoccupation croissantes, notamment dans les villes en forte expansion, car elle circonscrit la capacité des individus à accéder aux ressources spatiales et sociales. "L'individu est évalué sur sa faculté à se déplacer et à s'adapter. Cette montée de la mobilité comme exigence et comme valeur s'inscrit dans un large processus d'individuation » [Fol S., 2010, p. 52]. Une telle perspective conduit à interroger l'association entre mobilité et pauvreté [Le Breton, 2005 ; Fol S., 2009], au sens où le dénuement économique s'accompagne d'empêchements à se mouvoir hors de son environnement proche et par conséquent à accéder aux différentes aménités urbaines, à commencer par le travail. Ainsi, la mobilité spatiale, devenant une composante de la justice sociale, gagne un nouveau statut dans les études urbaines, au Nord comme au Sud. Cependant, cette valorisation de la mobilité, qui s'apparente parfois à une injonction [Bacqué M.-H., Fol S., 2007 ; Dureau F., Gouëset V., 2010] soulève bon nombre d'interrogations relatives à son analyse. D'une part, le rôle de la proximité et des ressources que celle-ci peut offrir [Fol S., 2010], est oblitéré au profit d'une valorisation de la capacité à circuler, envisagée comme moyen pour s'extraire d'une situation de pauvreté ou d'exclusion spatiale. D'autre part, cette importance accordée à la capacité de se mouvoir ne peut faire totalement abstraction des formes urbaines. Ainsi, le contexte de croissance et d'expansion spatiale rapides de certaines villes au Sud, notamment en Afrique de l'Ouest, associé à l'importance de l'informalité du travail et des modes d'habiter, conduisent à interroger de façon spécifique ce lien entre mobilités spatiales et justice sociale [Diaz Olvera, Plat, Pochet, 1998 ; Godard, 2002]. Enfin, une simple mesure de la fréquence ou de la durée des mobilités ne suffit pas à établir l'inégalité des

\footnotetext{
* Géographe, chargée de recherche à l'IRD (Institut de Recherche pour le Développement), UMR 205 " Migrations et société » (université Paris-Diderot, CNRS, IRD).

** Géographe, Professeur des universités, enseignant-chercheur en géographie, directeur de l'UMR 6590 ESO «Espaces et Sociétés » (université de Rennes 2, CNRS).

*** Démographe, directeur de recherche émérite à l'IRD (Institut de Recherche pour le Développement), UMR 201 «Sociétés et développement » (université de Paris 1 Panthéon-Sorbonne, IRD).
} 
individus face à cette ressource. Privilégier la fréquence ou la durée pour déceler les inégalités suppose que les déplacements intenses et parfois contraints sont préférables à ceux qui sont rares parce qu'ils ont été choisis. Une même pratique peut recouvrer des stratégies opposées [Korsu, Wenglenski, 2010] selon la localisation dans la ville, la capacité à se déplacer, la disposition d'un capital de mobilité [Delaunay, Fournier, 2014]. Et la "motilité », c'est-à-dire la capacité à se mouvoir selon Kaufmann [2005], ne signifie pas automatiquement la mise en œuvre d'un déplacement effectif. Elle peut correspondre à une maîtrise de l'espace urbain et des opportunités qu'il offre [Lannoy P., Ramadier T., 2007], mais pas obligatoirement à un processus d'inclusion sociale. Ainsi, l'analyse du lien entre mobilités et justice sociale ne peut faire abstraction non seulement des individus qui sont immobiles [Bergeon C. et al., 2013], mais aussi du sens des mobilités, celles-ci pouvant recouvrir autant des formes de différenciation que d'inégalités sociales et spatiales [Mignot, 2004].

Dans les villes de l'Afrique de l'Ouest et celles du Sahel en particulier, les inégalités sociales de la mobilité sont rarement débattues, même si l'on dispose de quelques études sur les déplacements quotidiens dans les capitales régionales ${ }^{1}$. Certes, l'étalement spatial est un phénomène qui a pris récemment une grande ampleur avec le processus de transition urbaine ; les conséquences de cet étalement sur la mobilité quotidienne se révèlent lentement. Cependant, celles-ci sont nuancées, car le peuplement de la ville n'est socialement pas homogène. Dans le cas de la capitale burkinabè, Ouagadougou, les périphéries qui se forment présentent le profil d'un habitat informel «non loti ${ }^{2}$, qui exprime une autre forme d'exclusion, aux côtés de nouveaux lotissements habités par les classes émergentes, les bénéficiaires des plus-values foncières réalisées dans le centre, voire les migrants internationaux de retour. De surcroît, la différenciation démographique des marges de la ville contribue à en complexifier le tissu social, avec l'arrivée de populations rajeunies, d'origine provinciale et plus fécondes. La ville de Ouagadougou est exemplaire de ces changements liés à la croissance des villes peu denses, mais millionnaires, dont l'accroissement rapide puise sa dynamique dans une transition démographique tardive, dans un pays à environ $70 \%$ rural, tandis que l'offre de transports collectifs reste notoirement insuffisante, ce qui explique partiellement la place singulière des véhicules à deux roues (vélos et motos) dans les déplacements quotidiens.

1. Pour Ouagadougou : l'enquête $\mathrm{I}_{\text {NRETS}}-L E T$ de 1992 [Diaz Olvera L., Plat D., 1996], celle du Bureau Cima+ International [2000], ainsi que les travaux du S ITRAss [2003].

2. À Ouagadougou, l'expression «non loti » désigne des quartiers autoconstruits illégalement, essentiellement périphériques, où les habitants ne disposent que de la propriété coutumière de leur parcelle. La particularité de la capitale burkinabè est que ces quartiers sont progressivement « lotis » par la municipalité, un acte qui permet à une grande partie des résidents informels d'accéder à des parcelles régularisées. Ainsi, construire sa maison dans un quartier non loti est devenu la stratégie la plus économique d'accès à la propriété pour les classes moyennes et populaires ; ces dernières comptent sur une régularisation, puis un équipement du quartier au bout de plusieurs années. 
La présente analyse de la mobilité quotidienne s'appuie sur une enquête sur le peuplement de l'ensemble de la ville de Ouagadougou, réalisée en $2009^{3}$. Cette étude démographique reste à ce jour la plus récente portant sur l'ensemble de la ville ${ }^{4}$. Elle s'est attachée à faire le lien entre démographie, logement et mobilité et elle offre la possibilité de questionner de nombreux aspects de la mobilité quotidienne à Ouagadougou, tels que les inégalités relatives au taux d'équipement en véhicules particuliers ou encore la hiérarchie des modes de transport utilisés par les habitants. D'autre part, l'enquête permet de voir en quoi la sédentarité ou les indicateurs de la mobilité quotidienne des individus (fréquence, durée, motifs des déplacements...) apportent des éléments pour apprécier les capacités inégales des individus à être mobiles et à construire un espace de vie leur permettant d'accéder aux ressources urbaines.

\section{Mesurer les mobilités spatiales à Ouagadougou}

Atteignant deux millions d'habitants en 2009 [Boyer F., Delaunay D., 2010] ${ }^{5}$, Ouagadougou reste une ville de taille modeste au regard d'autres capitales d'Afrique de l'Ouest, mais sa croissance démographique est élevée, avec un taux annuel moyen de 7,3\% entre 2005 et 2010 , de 7,2\% entre 2010 et 2015 et estimé à $6 \%$ entre 2015 et $2020^{\circ}$. La densité de peuplement reste pourtant faible (73,8 hab/ha), car la croissance démographique se projette en périphérie sans grandes contraintes physiques ou légales. Cet étalement spatial rapide est essentiellement informel, ce qui contribue à repousser toujours un peu plus loin les nouveaux candidats à la propriété [Prat M., 1996]. Même si ces quartiers sont à terme régularisés, une bonne partie de la population - $28 \%$ des habitants en 2009 - habite les quartiers non lotis, dépourvus d'infrastructures et éloignés du centre. Du fait des lenteurs du lotissement, ce mode d'extension urbaine contribue à une graduation des équipements de base (eau et électricité) en fonction de l'âge des quartiers, qu'ils soient lotis ou non.

L'enquête « Ouaga 2009 » a eu pour ambition de mesurer plusieurs formes de la mobilité spatiale à l'échelle de l'ensemble urbanisé ouagalais. Le choix d'un mode de sondage aréolaire, sur la base d'une définition in situ de l'espace urbanisé en 2009, produit des résultats représentatifs de l'ensemble de la ville de Ouagadougou. Dans un premier temps, nous avons réalisé une série de relevés GPS sur le terrain pour définir les limites de cet espace urbanisé, selon un critère de continuité et de type de bâti. Dans un second temps, et à des fins d'analyse, nous avons

\footnotetext{
3. « Ouaga 2009 : peuplement de Ouagadougou et développement urbain », projet coordonné par F. Boyer et D. Delaunay (IRD), financé par les Services de coopération de l'ambassade de France au Burkina Faso en 2009.

4. Le dernier recensement général de la population et de l'habitat a été effectué en 2006.

5. Sauf mention contraire, l'ensemble des données chiffrées est issu de l'enquête « Ouaga 2009 » [Boyer F., Delaunay D., 2010].

6. Source : World urbanization prospects, the 2014 revisions, population division, department of economic and social affairs, United Nations : https://esa.un.org/unpd/wup/DataQuery/ (consulté le 18 août 2017)
} 
découpé l'espace urbain en 65 polygones (carte 1) : ceux-ci se définissent par une certaine homogénéité de la morphologie et du bâti et ils ont été établis à la fois à partir d'observations fines de terrain et d'une analyse d'image satellite par Google Earth. Ces choix ont pour objectif de s'affranchir des limites administratives (limite communale et limites de secteurs) qui reflètent mal l'avancée de l'urbanisation.

Notre unité statistique primaire est le logement, pour privilégier les logiques de corésidence et qui aide à saisir les pratiques résidentielles complexes, ainsi que les arbitrages des occupants en matière de mobilité. L'enquête a porté sur 1965 logements, soit 10540 individus de tous âges. L'analyse repose sur un large éventail d'indicateurs relatifs aux individus, leur entourage, leur travail, l'équipement du logement. Chaque individu (âgé de 5 ans et plus) a été interrogé sur ses différentes pratiques de mobilité : à l'échelle de l'année, à celles de la journée et de la semaine, et à l'échelle biographique pour la moitié des individus de 18 ans et plus.

Plusieurs enquêtes antérieures existaient sur les mobilités quotidiennes à Ouagadougou. L'une des plus anciennes a été réalisée par l'INRETS et le laboratoire LET en 1992 [Diaz Olivera L., Plat D., 1996] ; l'autre en 2000, par le Bureau Cima+ International. Une décennie plus tard, l'originalité de l'étude «Ouaga 2009 » [Boyer F., Delaunay D., 2010] a étudié non seulement les déplacements au quotidien, mais aussi les pratiques hebdomadaires des habitants âgés de cinq ans et plus ; deux temporalités qui seront présentées et analysées ici. L'échelle quotidienne se focalise sur les déplacements proprement dits de la journée précédant l'enquête. Est privilégié l'aspect «technique » : la fréquence, la durée, le mode de transport et la destination du déplacement. Cependant, cette échelle du quotidien ne se rapporte qu'aux mobilités effectives, sans prendre en compte les sédentaires. Pour cette raison, et pour couvrir un spectre plus large de motifs, une autre observation a été entreprise à l'échelle de la semaine ${ }^{7}$. Elle privilégie les pratiques individuelles (vie sociale, activités liées à l'activité domestique, économique...). Les statistiques sont analysées pour les individus, dont les sorties hebdomadaires sont agrégées, en rapport avec leurs caractéristiques.

\section{Ouagadougou, une ville où l'on se déplace surtout à pied, à vélo ou à « moto " $^{8}$}

L'analyse qui suit porte sur les déplacements réalisés par les Ouagalais de plus de 5 ans, au cours de la journée précédant l'enquête, et ignore les habitants qui ne sont pas sortis de chez eux. L'accent est mis sur les modes de transport,

\footnotetext{
7. La semaine est définie comme les sept jours précédant la date de l'enquête. Les week-ends sont compris, mais les données ne permettent pas de les distinguer des jours ouvrables dans les traitements.

8. Terme générique qui désigne à Ouagadougou l'ensemble des deux-roues motorisés, sans distinction de taille ou de puissance. Il sera utilisé dans ce sens tout au long du texte.
} 
l'équipement des particuliers et les motifs de chacun des trajets. Dans la capitale, où l'offre de transports en commun restait embryonnaire au moment de l'enquête, l'essentiel des trajets quotidiens est réalisé en véhicules particuliers ou à pied. La possession d'un moyen de transport est la première source de différenciation des mobilités et des inégalités d'accès à la ville. La capitale burkinabè se singularise, à l'échelle régionale, par la distribution modale des déplacements quotidiens, avec la modestie des transports en commun et la part élevée des véhicules à deux roues. Ainsi, $54 \%$ des trajets quotidiens se font à pied, $21 \%$ en vélo, $20 \%$ en moto et $3 \%$ en voiture (comme conducteur ou passager) tandis que les transports collectifs (autobus, taxis collectifs) ne représentent que $1 \%$ du total. En dehors de la marche, quasi exclusive chez les enfants et qui perd ensuite de son importance relative avec l'âge, Ouagadougou est avant tout une ville de conducteurs de motos et de bicyclettes. Ainsi, elle se singularise par rapport à d'autres capitales comme Dakar, Conakry ou Niamey, où la marche représente autour de $70 \%$ ou plus des déplacements [Diaz Olvera et al., 2010].

\section{Des transports collectifs historiquement insuffisants}

Ouagadougou se distingue par l'extrême rareté des transports collectifs, qu'ils soient publics (les bus de la SOTRACO) ou privés (les taxis collectifs). Ces deux modes de transport combinés ne représentent que $1 \%$ des déplacements quotidiens (cf. : Tableau 1). À l'opposé, les autres capitales d'Afrique de l'Ouest, comme Bamako, Niamey ou Cotonou, mettent à la disposition des citadins une offre pléthorique de transports formels ou informels (taxis individuels et collectifs, minibus, motos taxis). Comme l'a signalé S. Bamas [2003, p. 64], l'absence de transports collectifs informels à Ouagadougou est en grande partie l'héritage d'un choix politique de la révolution sankariste, qui était d'empêcher le développement des transports artisanaux (minibus et taxis collectifs), afin de placer la régie des transports en commun en situation de monopole, pour garantir sa rentabilité économique. À cela s'ajoute, à la même époque, une incitation des pouvoirs publics à utiliser les vélos qui avaient le double avantage d'être fabriqués localement et de diminuer la facture énergétique du pays [Diaz Olvera, Plat, Pochet, 1998, p. 70]. Par la suite, cette situation unique en Afrique de l'Ouest s'est renforcée : la faiblesse des transports en commun a contraint les ménages à s'équiper en deux-roues et la prolifération de ces derniers a limité en retour la demande pour les transports collectifs. 
Tableau 1 - Distribution par mode de transport des déplacements quotidiens des individus de 5 ans et plus, selon le sexe, l'âge, le type d'activités et la localisation du logement (2009) ${ }^{9}$

\begin{tabular}{|c|c|c|c|c|c|c|c|}
\hline & \multicolumn{3}{|c|}{$\begin{array}{cc}\text { Marche Bicyclette } & \text { Deux- } \\
\text { à pied } & \text { roues à } \\
\text { moteur }\end{array}$} & \multicolumn{2}{|c|}{$\begin{array}{l}\text { Voiture Taxi } \\
\text { collectif }\end{array}$} & \multicolumn{2}{|c|}{ Bus Autre } \\
\hline \multicolumn{8}{|c|}{ Distribution selon le sexe (en \%) } \\
\hline Homme & 47 & 23 & 24 & 4 & 1 & 0 & 1 \\
\hline Femme & 63 & 18 & 15 & 2 & 1 & 0 & 0 \\
\hline \multicolumn{8}{|c|}{ Distribution selon la classe d'âge (en \%) } \\
\hline $5-11$ ans & 85 & 9 & 4 & 2 & 0 & 0 & 0 \\
\hline $12-19$ ans & 68 & 25 & 6 & 1 & 0 & 0 & 0 \\
\hline $20-29$ ans & 43 & 27 & 26 & 2 & 1 & 0 & 0 \\
\hline $30-44$ ans & 30 & 21 & 40 & 7 & 1 & 1 & 1 \\
\hline $45-59$ ans & 37 & 19 & 31 & 9 & 2 & 1 & 1 \\
\hline 60 ans et + & 57 & 18 & 14 & 9 & 1 & 0 & 1 \\
\hline \multicolumn{8}{|c|}{ Distribution selon le type d'activité (en \%) } \\
\hline Salarié du secteur public & 10 & 5 & 68 & 16 & 0 & 0 & 0 \\
\hline Salarié du secteur privé & 36 & 20 & 34 & 8 & 2 & 1 & 0 \\
\hline Employeur/ patron & 18 & 21 & 36 & 22 & 0 & 0 & 2 \\
\hline Indépendant avec local & 39 & 22 & 33 & 3 & 0 & 0 & 2 \\
\hline Indépendant sans local & 46 & 33 & 19 & 1 & 2 & 0 & 0 \\
\hline Aide familial/apprenti & 52 & 30 & 14 & 2 & 1 & 0 & 1 \\
\hline Journalier & 41 & 42 & 15 & 0 & 1 & 1 & 0 \\
\hline Inactif pour études & 54 & 25 & 19 & 2 & 0 & 0 & 0 \\
\hline Inactifs autre & 53 & 22 & 18 & 3 & 3 & 1 & 0 \\
\hline Actif au chômage & 52 & 23 & 23 & 1 & 0 & 1 & 0 \\
\hline Actif indéterminé & 49 & 16 & 32 & 3 & 0 & 0 & 0 \\
\hline Activités domestiques & 69 & 17 & 10 & 1 & 2 & 1 & 1 \\
\hline $\begin{array}{l}\text { Moins de } 12 \text { ans non } \\
\text { scolarisé }\end{array}$ & 85 & 9 & 4 & 2 & 0 & 0 & 0 \\
\hline \multicolumn{8}{|c|}{ Distribution selon la localisation du logement (en \%) } \\
\hline Quartiers lotis avant 1980 & 56 & 7 & 28 & 7 & 0 & 1 & 0 \\
\hline $\begin{array}{l}\text { Q. lotis entre } 1983 \\
\text { et la fin des années } 1990\end{array}$ & 52 & 16 & 26 & 4 & 1 & 0 & 0 \\
\hline Q. lotis dans années 2000 & 61 & 24 & 12 & 1 & 0 & 1 & 1 \\
\hline Quartiers non lotis denses & 50 & 35 & 13 & 0 & 1 & 0 & 1 \\
\hline $\begin{array}{l}\text { Quartiers non lotis peu } \\
\text { denses }\end{array}$ & 57 & 33 & 7 & 0 & 0 & 0 & 2 \\
\hline Total & 54 & 20 & 20 & 3 & 1 & 0 & 0 \\
\hline
\end{tabular}

Source : Enquête « Ouaga $2009 »$

9. Les calculs ont été réalisés à partir du calendrier journalier : est donc pris en compte l'ensemble des déplacements réalisés par les individus de 5 ans et plus au cours de la journée précédant l'enquête. 
Cette politique n'a pourtant pas permis l'essor d'une offre suffisante de transports publics : trois entreprises se sont succédé au cours des 20 dernières années ${ }^{10}$, sans jamais parvenir à équilibrer leurs comptes ni à couvrir les besoins des Ouagalais. Début 2010, sur les 14 lignes officielles de la SOTRACO, 9 seulement étaient opérationnelles et six ans plus tard, en août 2016, 11 lignes seulement étaient en service, de façon irrégulière, ne couvrant que le centre-ville et les proches périphéries. Historiquement, le parc roulant de l'entreprise, vétuste et très insuffisant, n'assurait pas la continuité du service, d'autant que la demande concentrée sur quatre pics de fréquentation quotidiens (autour de 7 heures, 12 heures, $14 \mathrm{~h} 30$ et 18 heures) ne permettait pas de remplir les bus en heures creuses, alors qu'elle dépassait au contraire la capacité opérationnelle de la SOTRACO en heure de pointe [CIMA+, 2000, p. 5]. Au moment de l'enquête Ouaga 2009, la situation de l'entreprise restait donc très difficile : elle offrait un service de qualité médiocre, avec une desserte incertaine et un temps moyen de déplacement de 42,5 minutes de porteà-porte (deux fois plus qu'avec les autres modes de transport). Enfin, elle ne couvrait pas ses frais de production, en raison d'un taux de remplissage des bus trop faible ${ }^{11}$. Sept ans plus tard, la qualité du service ne s'est pas améliorée de façon significative, malgré l'acquisition d'une cinquantaine de bus, ce qui porte en août 2016 le parc roulant à 78 bus, dont une quarantaine seulement circule de façon régulière ${ }^{12}$.

\section{Un taux d'équipement en moyens de transport individuels} relativement élevé

À Ouagadougou, se rendre rapidement à une destination plus ou moins lointaine implique de disposer d'un moyen de transport individuel, le plus souvent une bicyclette, ou mieux une moto. Six habitants sur dix de 12 ans et plus possèdent un moyen de transport (une proportion qui s'élève à $68 \%$ entre 20 et 59 ans, c'est-à-dire au cours de la vie active), ce qui facilite l'autonomie des déplacements pour les populations concernées, alors que pour les autres, les plus jeunes et les plus âgés notamment, la quasi-absence de transports collectifs limite singulièrement leur mobilité quotidienne. Dans le détail, un cinquième seulement de la population ouagalaise de 12 ans et plus possède une moto et/ou une voiture et un tiers, une bicyclette. Bien que les transports individuels motorisés restent un privilège réservé à une minorité d'Ouagalais, la capitale burkinabè se distingue des autres capitales d'Afrique de l'Ouest, où l'équipement en moyen de transport individuel est extrêmement réduit (proche de zéro par exemple à Dakar ou Conakry pour ce qui est des motos).

10. La RNTC X9, entreprise publique créée durant les années 1980 a été liquidée à partir de 1994 et remplacée en 1996 par une société mixte, la $\mathrm{S}_{\text {OTRAo }}$ (Société de transports de Ouagadougou), qui a fait faillite en 2002. L'entreprise actuelle, la S S pris la relève en 2003, après plus d'un an d'interruption totale du service.

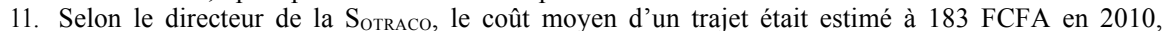
alors que le tarif appliqué n'était que de 150 FCFA (un montant toujours en vigueur en août 2016).

12. Sources : entretiens auprès du directeur de la $\mathrm{S}_{\text {Otraco }}$ en juin 2009 et janvier 2010 et entretien réalisé par Seydou Séré (doctorant, Université de Rennes 2) auprès du directeur d'exploitation de la $\mathrm{S}_{\text {OtRACO }}$ en août 2016. 
Une mesure de l'équipement à l'échelle des logements permet de nuancer ces chiffres individuels et de donner une appréciation plus collective du déficit d'équipement de certains : un logement sur dix (12\%) n'est doté d'aucun moyen de transport, ce qui contraint ses résidents soit à la marche, soit à dépendre des transports en commun, soit enfin à dépendre de l'entraide des personnes disposant d'un véhicule personnel. Un tiers des logements n'a pas de vélo, plus de la moitié (55\%) ne possède pas de moto, $94 \%$ pas de voiture. Les « usagers apparentés », selon la formule de Diaz Olvera et Plat [1997, p. 213-225], c'est-à-dire les personnes partageant des véhicules motorisés ou pratiquant le covoiturage sont peu nombreux en raison du faible équipement des logements.

Le taux d'équipement en vélos des femmes est 1,4 fois plus faible que celui des hommes ; un rapport qui passe à 2,1 pour les motos et à 2,7 pour les voitures (tableau 2). L'âge des individus, ainsi que l'étape dans le cycle de vie apparaissent décisifs. Les plus jeunes, aux âges de la scolarisation, se révèlent les plus pénalisés, étant sans moyens ou non motorisés. C'est une situation très discriminante pour ceux qui résident dans les quartiers périphériques, où l'implantation d'établisse- ments scolaires publics du secondaire est inexistante, au mieux peu dense. Cette exclusion dans l'accès à un mode de transport se poursuit jusqu'à l'entrée dans la vie active, puisqu'elle concerne également les 20-24 ans, même si leur situation tend à s'améliorer légèrement, comparativement à leurs cadets. Ainsi, la poursuite d'études supérieures, comme la recherche d'un emploi, peut se heurter à ce déficit d'équipement. Progressivement, à partir de 25 ans et jusque vers l'âge de 55 ans, les hommes sont de mieux en mieux équipés en moyens de transport motorisés. Enfin, pour les générations âgées, la dépossession s'accentue. La catégorie socio- professionnelle accompagne ce mouvement : les activités domestiques, réservées aux femmes, les aides familiaux et les apprentis, catégories qui renvoient à l'entrée dans la vie active, de même que les catégories les plus précaires du secteur informel (journaliers, indépendants sans local) n'ont que faiblement accès à un mode de transport individuel (hormis le vélo pour la dernière catégorie). Les salariés du secteur public et les employeurs ou patrons sont mieux dotés que les indépendants avec local ou les salariés du secteur privé.

Finalement, l'inégale possession d'un moyen de transport individuel, tout comme l'inégale qualité des moyens de transport, dans un contexte de pénurie des transports collectifs, renforce les différenciations subies par les femmes et les jeunes, notamment les jeunes adultes. Elle touche également les périphéries où les jeunes couples sont contraints de s'installer, même si ce handicap est partiellement compensé par un taux d'équipement en bicyclette nettement supérieur à la moyenne dans les périphéries non loties. La formation des jeunes adultes s'en trouve freinée, comme l'accès au marché du travail des jeunes actifs, particulièrement pour les femmes. 
Tableau 2 - Taux d'équipement des individus de 12 ans et plus $\left(^{*}\right)$ en moyens de transport particulier

\begin{tabular}{|c|c|c|c|c|c|}
\hline & \multirow{2}{*}{$\begin{array}{l}\text { Possession } \\
\text { d'un moyen } \\
\text { de transport } \\
\text { ou }+\end{array}$} & \multicolumn{4}{|c|}{ Dont : } \\
\hline & & Bicyclette & Mot & oiture & Autre \\
\hline \multicolumn{6}{|c|}{ Possession d'un moyen de transport selon le sexe (en \%) } \\
\hline Hommes & 74 & 39 & 28 & 3 & 4 \\
\hline Femmes & 45 & 28 & 13 & 1 & 2 \\
\hline \multicolumn{6}{|c|}{ Distribution selon la classe d'âge (en \%) } \\
\hline $12-19$ ans & 38 & 33 & 3 & 0 & 2 \\
\hline $20-29$ ans & 63 & 38 & 21 & 0 & 3 \\
\hline $30-44$ ans & 76 & 32 & 36 & 4 & 4 \\
\hline $45-59$ ans & 67 & 29 & 28 & 7 & 4 \\
\hline 60 ans et + & 48 & 28 & 16 & 4 & 0 \\
\hline \multicolumn{6}{|c|}{ Possession d'un moyen de transport selon la catégorie socioprofessionnelle (en \% } \\
\hline Salarié du secteur public & 95 & 6 & 69 & 14 & 6 \\
\hline Salarié du secteur privé & 68 & 31 & 30 & 4 & 3 \\
\hline Employeur/patron & 86 & 23 & 37 & 26 & 0 \\
\hline Indépendant avec local & 71 & 39 & 28 & 2 & 1 \\
\hline Indépendant sans local & 68 & 48 & 18 & 0 & 1 \\
\hline Aide familial/apprenti & 54 & 41 & 10 & 0 & 3 \\
\hline Journalier & 72 & 53 & 16 & 0 & 2 \\
\hline Inactif pour études & 55 & 38 & 15 & 0 & 2 \\
\hline Inactifs autre & 45 & 22 & 15 & 4 & 4 \\
\hline Actif au chômage & 53 & 30 & 22 & 0 & 1 \\
\hline Actif indéterminé & 54 & 22 & 21 & 2 & 8 \\
\hline Activités domestiques & 33 & 24 & 7 & 0 & 2 \\
\hline \multicolumn{6}{|c|}{ Possession d'un moyen de transport selon le type de quartier (en \%) } \\
\hline Quartiers lotis avant 1983 & 46 & 13 & 24 & 5 & 4 \\
\hline $\begin{array}{l}\text { Quartiers Lotis entre } 1983 \text { et la } \\
\text { fin des } 90^{\prime}\end{array}$ & 59 & 27 & 27 & 3 & 2 \\
\hline $\begin{array}{l}\text { Quartiers lotis pendant les } \\
\text { années } 2000\end{array}$ & 62 & 42 & 16 & 1 & 3 \\
\hline Quartiers non lotis denses & 69 & 53 & 14 & 0 & 3 \\
\hline Quartiers non lotispeu denses & 57 & 46 & 7 & 0 & 4 \\
\hline $\begin{array}{l}\text { Total } \\
\text { (*) Plus de } 18 \text { ans pour les } \\
\text { voitures }\end{array}$ & 60 & 34 & 21 & 2 & 3 \\
\hline
\end{tabular}

Source : Enquête « Ouaga 2009 » 


\section{Une " hiérarchie modale » marquée par des divisions socio-économiques}

Pour reprendre la formule employée dans une étude précédente [Diaz Olvera, Plat, Pochet, 1998, P. 103-105], la « hiérarchie modale » est avant tout une « hiérarchie sociale ». L'enquête « Ouaga 2009 » confirme cet aspect (tableau 1) sur une analyse fine des catégories socio-économiques, incluant les inactifs. Ainsi la marche est un mode de déplacement minoritaire chez les salariés du secteur public et chez les employeurs ou patrons (10 et $18 \%$ des déplacements), alors qu'elle est massive pour les autres catégories. Les inactifs (scolarisés, enfants de moins de 12 ans, personnes dévolues aux tâches domestiques, etc.), comme les actifs précaires, les apprentis, les aides familiaux ou les chômeurs effectuent plus de $50 \%$ de leurs déplacements quotidiens à pied. À l'inverse, la voiture apparaît comme le mode de transport le plus exclusif, accessible uniquement aux deux catégories socioprofessionnelles les plus favorisées, les salariés du public et les employeurs et patrons. Les déplacements à moto sont mieux répartis entre toutes les catégories professionnelles, même si les salariés du secteur public apparaissent une fois encore mieux dotés que les autres. Enfin, les déplacements à bicyclette sont surtout le fait des journaliers (qui réalisent ainsi $42 \%$ de leurs déplacements) : cette association à la précarité, à une résidence en périphérie également (carte 1), conforte la perception socialement déclassante des cyclistes à Ouagadougou.

Aux effets de la catégorie socioprofessionnelle s'ajoute un celui de l'âge, beaucoup plus notable pour l'usage du moyen de transport que pour la seule observation du taux d'équipement. Effectivement, alors que l'usage de la bicyclette, comme celui de la moto, s'établit autour de $26 \%$ des déplacements pour les 20-29 ans, $40 \%$ des déplacements des 30-44 ans s'effectuent à moto et $7 \%$ des déplacements en voiture. Pour les classes d'âges supérieures, l'usage de la moto diminue comme celui de la bicyclette, au profit de la voiture (qui reste marginale) et de la marche, surtout pour les plus de 60 ans. Ainsi, l'amélioration du niveau de vie du logement lorsque l'individu avance en âge s'accompagne d'une amélioration des conditions de déplacement, hormis pour les plus âgés qui utilisent moins les deux-roues, sans doute en raison de l'inconfort et du danger qu'ils représentent. Cependant, ces personnes bénéficient des localisations résidentielles les plus centrales et ont des habitudes de déplacement plus restreintes.

Les inégalités en matière de mobilité quotidienne s'expriment également par le genre des utilisateurs, déjà remarqué dans d'autres études. Il découle en partie de l'inégale insertion des femmes et des hommes sur le marché de l'emploi : le taux d'activité des individus âgés de 12 ans et plus est de $70 \%$ pour les hommes, alors qu'il n'est que de $42 \%$ pour les femmes. Le fait que nombre de femmes travaillent à domicile contribue aussi à ce phénomène. Les inégalités de genre se retrouvent également dans la distribution modale des déplacements. Déjà en 1992, Diaz et Plat [1997 : 213-225] parlaient d'une logique de « confiscation » des véhicules motorisés par les hommes au sein des familles. Cette logique s'applique toujours en 2009, puisque les hommes utilisent plus les véhicules particuliers que les femmes (y compris le vélo), tandis que ces dernières recourent davantage à la 
Carte 1 - Se déplacer à vélo à Ouagadougou

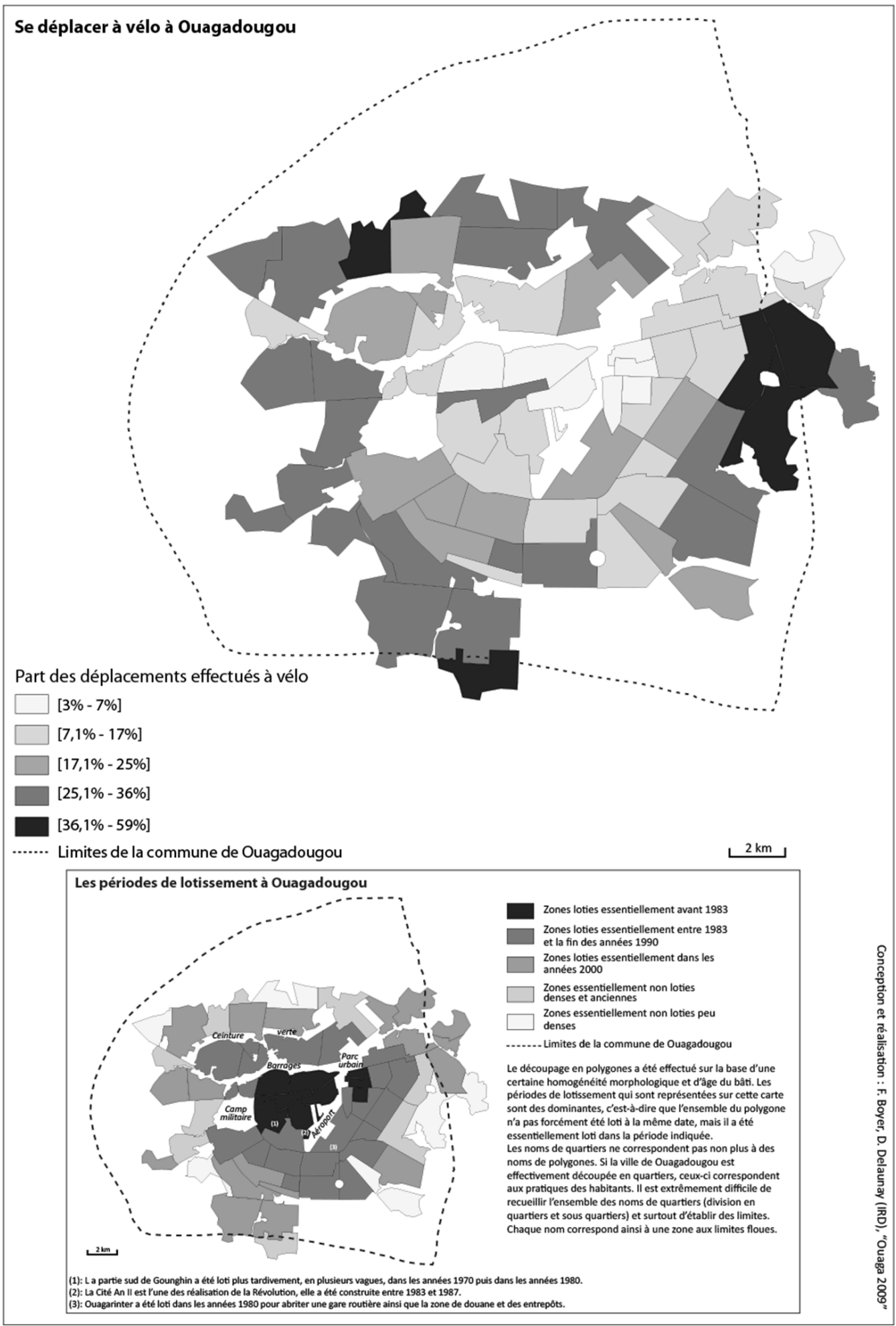

Sources : Enquête « Ouaga 2009» 
marche, au taxi collectif et au bus (cf. : Tableau 2). Cette tendance se maintient dans le temps : en 2000, la marche représentait $42 \%$ des déplacements pour l'ensemble de la population, mais $56 \%$ pour les femmes [Cusset, 1997].

Dans le contexte ouagalais de pénurie des transports publics, il est logique que la hiérarchie modale reflète celle, socio-économique, de la possession privative quasi exclusive des moyens de transport : obtenir un emploi conditionne, voire précède, l'acquisition de la mobilité. Cependant, il est à craindre que l'absence d'une offre de transports collectifs pénalise l'avenir de l'économie urbaine dans la mesure où cette absence est préjudiciable aux jeunes scolarisables, aux nouveaux candidats sur le marché du travail comme aux femmes. Or la jeunesse de la population alimentée par la migration vers la capitale - malgré un recul de la natalité et de la mortalité - contribue à étendre la ville ; les femmes libérées progressivement des tâches reproductives s'impliquent dans les activités rémunérées. Pour ces forces vives qui se voient éloignées du centre, la possibilité de se déplacer est contrariée par la pénurie de transports collectifs et le faible équipement individuel qui contribuent à leur précarité professionnelle.

\section{Des pratiques de mobilité adaptées à la ville et à l'équipement}

La pratique de la ville par les mobilités s'inscrit dans un faisceau de contraintes et de choix individuels, parmi lesquels se retrouvent autant les priorités occupationnelles, que la capacité à utiliser ou non les ressources urbaines. Par ailleurs, au sein de l'unité résidentielle, les individus procèdent à des arbitrages qui déterminent l'ampleur et l'intensité de leurs déplacements quotidiens. Ainsi, une partie des individus peut être sédentaire momentanément ou pour certains motifs de déplacements, alors que d'autres vont présenter des profils d'hypermobilité également variables dans le temps et selon les motifs.

La diversité des pratiques de mobilité quotidienne est enregistrée selon le motif de sortie du logement, la durée, la fréquence et le mode de transport. Ces sorties ont été relevées pour la semaine précédant le passage du questionnaire. Pour l'analyse, une catégorisation simplifiée des motifs a été reconstruite, distinguant les études, le travail, la vie sociale (loisirs, pratiques religieuses, visites) et les activités liées à la vie domestique (achats courants et rares, soins de santé). Ces mesures sont agrégées pour l'individu; la somme des sorties de la semaine par motif est rapportée au nombre de personnes ( +5 ans) de la catégorie. Contrairement aux déplacements des seules personnes mobiles, la population de référence inclut celles qui ne se sont pas sorties pour ce motif durant la semaine.

La pratique urbaine par les mobilités est abordée du point de vue des individus (et non plus des trajets); elle donne à voir le rapport des habitants avec la ville par le biais de ce qu'ils y font. La question des inégalités se pose alors non plus en termes des moyens, durées du déplacement, mais de la capacité à vivre la ville, y travailler, consommer, avoir une vie sociale. Le groupe des personnes qui n'accèdent à aucune de ces fonctions révèle une situation extrême de rupture avec leur environnement. 
Tableau 3 - Pratiques de mobilité (exprimées par la somme des sorties individuelles de la semaine) selon le motif, le sexe, la classe d'âge, la catégorie socioprofessionnelle, la localisation résidentielle et le moyen de transport (individus de 5 ans et plus)

\begin{tabular}{|c|c|c|c|c|c|}
\hline & $\begin{array}{c}\text { Fréquence } \\
\text { - Motif } \\
\text { Travail }\end{array}$ & $\begin{array}{l}\text { Fréquence } \\
\text { - Motif } \\
\text { Études }\end{array}$ & $\begin{array}{c}\text { Fréquence } \\
\text { - Motif } \\
\text { Consommation }\end{array}$ & $\begin{array}{l}\text { Fréquence } \\
\text { - Motif } \\
\text { Vie } \\
\text { sociale }\end{array}$ & $\begin{array}{c}\text { Fréquence } \\
\text { - Tous } \\
\text { motifs }\end{array}$ \\
\hline \multicolumn{6}{|c|}{ Distribution selon le sexe } \\
\hline Homme & 3,3 & 1,8 & 0,9 & 4,3 & 10,3 \\
\hline Femme & 1,6 & 1,8 & 2,1 & 2,5 & 8 \\
\hline \multicolumn{6}{|c|}{ Distribution selon la classe d'âge } \\
\hline $5-9$ ans & 0,2 & 5,4 & 0,3 & 2,8 & 8,7 \\
\hline $10-19$ ans & 1,4 & 3,3 & 1,6 & 2,8 & 9,1 \\
\hline $20-29$ ans & 3,1 & 0,9 & 2 & 3,8 & 9,7 \\
\hline $30-39$ ans & 4 & 0,3 & 1,9 & 3,1 & 9,2 \\
\hline $40-49$ ans & 3,9 & 0 & 1,5 & 3,6 & 9 \\
\hline $50-59$ ans & 3,2 & 0 & 0,9 & 4 & 8,1 \\
\hline $60-69$ ans & 1,9 & 0 & 0,7 & 5,4 & 7,9 \\
\hline 70 ans et + & 1,2 & 0 & 0,7 & 3,8 & 5,8 \\
\hline \multicolumn{6}{|c|}{ Distribution selon le type d'activité } \\
\hline $\begin{array}{l}\text { Salarié du secteur } \\
\text { public }\end{array}$ & 5,7 & 0,3 & 1,1 & 3,6 & 10,7 \\
\hline $\begin{array}{l}\text { Salarié du secteur } \\
\text { privé }\end{array}$ & 5,8 & 0,3 & 1,4 & 2,8 & 10,2 \\
\hline $\begin{array}{l}\text { Salarié dans le } \\
\text { logement }\end{array}$ & 1,4 & 0,3 & 3,3 & 2,1 & 7,1 \\
\hline Employeur/patron & 5,7 & 0 & 1,4 & 2,6 & 9,7 \\
\hline $\begin{array}{l}\text { Indépendant avec } \\
\text { local }\end{array}$ & 5,2 & 0,1 & 1,4 & 4,1 & 10,8 \\
\hline $\begin{array}{l}\text { Indépendant sans } \\
\text { local }\end{array}$ & 5,3 & 0,1 & 1,4 & 3,3 & 10,1 \\
\hline $\begin{array}{l}\text { Indépendant dans le } \\
\text { logement }\end{array}$ & 1,2 & 0,1 & 2,1 & 3,2 & 6,6 \\
\hline $\begin{array}{l}\text { Indépendant/aide } \\
\text { familial dans } \\
\text { l'agriculture }\end{array}$ & 2,8 & 0,1 & 0,8 & 4,8 & 8,5 \\
\hline Journalier & 5,6 & 0,1 & 1 & 5,4 & 12,2 \\
\hline Apprenti & 5,7 & 0,4 & 1,1 & 2,4 & 9,6 \\
\hline Aide familial & 5,4 & 0,3 & 1,1 & 4,1 & 11 \\
\hline Activités domestiques & 0,3 & 0,2 & 3,4 & 2,6 & 6,4 \\
\hline
\end{tabular}




\begin{tabular}{|c|c|c|c|c|c|}
\hline Moins de 12 ans & 0,2 & 5,3 & 0,5 & 2,8 & 8,7 \\
\hline $\begin{array}{l}\text { Étudiant de } 12 \text { ans et } \\
\text { plus }\end{array}$ & 0,3 & 5,3 & 1,2 & 3,5 & 10,3 \\
\hline Chômage & 0,3 & 0,1 & 1,1 & 5,8 & 7,3 \\
\hline Autres inactifs/NSP & 0,7 & 0,9 & 1,7 & 4,4 & 7,8 \\
\hline \multicolumn{6}{|c|}{ Distribution selon la localisation du logement } \\
\hline $\begin{array}{l}\text { Quartiers lotis avant } \\
1980\end{array}$ & 2,4 & 1,7 & 2 & 3,1 & 9,2 \\
\hline $\begin{array}{l}\text { Q. lotis entre } 1983 \text { et } \\
\text { la fin des années } 1990\end{array}$ & 2,4 & 1,9 & 1,7 & 3,9 & 9,9 \\
\hline $\begin{array}{l}\text { Q. lotis dans } \\
\text { années } 2000\end{array}$ & 2,5 & 1,9 & 1,3 & 3,1 & 8,8 \\
\hline $\begin{array}{l}\text { Quartiers non lotis } \\
\text { denses }\end{array}$ & 2,8 & 1,6 & 1,2 & 3 & 8,6 \\
\hline $\begin{array}{l}\text { Quartiers non lotis } \\
\text { peu denses }\end{array}$ & 2,2 & 1,5 & 0,8 & 2,2 & 6,7 \\
\hline \multicolumn{6}{|c|}{ Distribution selon la possession d'un moyen de transport } \\
\hline $\begin{array}{l}\text { Possède un moyen } \\
\text { de transport }\end{array}$ & 3,5 & 1,3 & 1,4 & 3,8 & 10 \\
\hline $\begin{array}{l}\text { Ne possède pas } \\
\text { de moyen de transport }\end{array}$ & 1,4 & 2,3 & 1,6 & 2,9 & 8,2 \\
\hline
\end{tabular}

Source : Enquête « Ouaga 2009 »

\section{La sédentarité absolue}

Il existe des sédentarités pour tel motif (qui n'a pas eu de vie sociale extérieure par exemple) et une sédentarité absolue quand la personne interrogée n'est pas du tout sortie de chez elle et n'a de fait eu aucune pratique de la ville. Cette «noncitadinité » est rare, seuls $5 \%$ des habitants de 5 ans et plus se déclarent dans cette situation. Cette immobilité peut cacher une privation de certaines activités faute d'une capacité suffisante à se déplacer, variable au cours du cycle de vie et selon les motifs. Très jeunes, ils sont légèrement plus nombreux (6\% des 5-9 ans) qu'au cours de la vie active. Ce taux de sédentarité est stable entre 10 et 49 ans $(4 \%)$ pour remonter régulièrement ensuite, avec une envolée à partir de 60 ans (19\% des 60-69 ans) et plus radicalement après 70 ans. À partir de cet âge, $43 \%$ déclarent avoir été sédentaires la semaine précédente, $34 \%$ pour les hommes, $52 \%$ pour les femmes. De façon attendue, les hommes aux âges de l'activité sont rarissimes, entre 2 et $4 \%$ et sont essentiellement des personnes handicapées. Pour ce qui est des femmes, la sédentarité diminue légèrement en fin de vie féconde (elle passe de $5 \%$ entre 20 et 39 ans à $3 \%$ entre 40 et 49 ans), ce qui correspond à la fin du cycle reproductif et à un accroissement de l'activité rémunérée. Ainsi, entre 30 et 49 ans, hommes et femmes sont concernés à l'identique. Alors que l'immobilité se maintient à un bas niveau pour les hommes entre 50 et 59 ans (3\%), elle augmente pour les femmes à la même tranche d'âge, passant à $17 \%$. 
Une hypothèse est qu'à cet âge, les femmes subissent moins certaines mobilités contraintes, telles celles liées à la consommation; elles peuvent être remplacées dans cette tâche par leurs filles plus jeunes. Également, le travail à domicile, domestique ou informel est plus fréquent chez les femmes que chez les hommes. Par conséquent, les différences genrées restent très limitées aux âges actifs, ne prenant de l'ampleur qu'à l'aune de la vieillesse.

Le statut socio-économique fait reculer le risque d'avoir été immobile, qui se réduit avec l'ascension sociale, sans qu'apparaisse une différence de genre à statut équivalent. Les métiers plus qualifiés obligent à se rendre sur un lieu spécifique de travail et donc à disposer d'un moyen de transport. Les salariés comme les indépendants présentent des choix de sédentarité très rares, y compris lorsque le travail se réalise au domicile. Cela vaut aussi pour les catégories plus précaires, journaliers, apprentis ou aides familiaux, également contraints par l'activité. La possession d'un moyen de transport, quant à elle, réduit sensiblement l'immobilité, sans être véritablement discriminante ; les hommes possédant un moyen de transport présentent un taux de sédentarité absolue de $2 \%$, ceux qui n'en ont pas de $7 \%$ et pour les femmes, ce taux passe respectivement de $4 \%$ à $8 \%$.

Ce qui pourrait être interprété comme une discrimination forte est donc de faible portée. En effet, la socialisation par des visites aux parents (qui touchent deux personnes sur dix) est en retrait en raison de leur dispersion dans la ville, tandis que les visites aux amis, qui concernent $27 \%$ des Ouagalais, sont plus souvent pratiquées par les hommes, les jeunes, dans un rayon proche du logement.

\section{Les pratiques hebdomadaires}

L'analyse des pratiques de mobilité à l'échelle de la semaine, qui concernent l'ensemble des individus, ne fait pas apparaître de différenciations fortes ; en effet, alors que les hommes sortent en moyenne 10,3 fois de leur logement chaque semaine, les femmes sortent 8 fois, et ce quel que soit le motif. Tout se passe comme si chaque individu parvient à maintenir un rapport commun et partagé à la ville, quel que soit le genre, l'âge, le quartier de résidence ou même la catégorie socioprofessionnelle. Si des différenciations apparaissent, elles relèvent des contraintes liées aux rôles sociaux des uns et des autres (le travail pour les hommes, faire les courses pour les femmes, s'éduquer pour les plus jeunes...). Ainsi, pour les hommes, le travail et la vie sociale affectent en priorité leurs pratiques hebdomadaires ; les femmes étant, quant à elles, plus concernées par les motifs de consommation que les hommes.

Il en est de même pour ce qui est de la distribution en fonction de la classe d'âge. À partir de 20 ans, le travail se substitue aux études pour les mobilités contraintes, alors que la vie sociale occupe une place de plus en plus importante. À l'échelle du cycle de vie, tout se passe comme si l'individu disposait d'une mobilité globalement stable avant la vieillesse, l'arbitrage s'effectuant selon les motifs dans le cadre d'un budget stable de sorties. Pour les femmes, un net 
décrochage s'observe à plus de 70 ans (3,8 sorties par semaine, 8 pour les hommes) alors qu'entre 5 et 69 ans, l'intensité des pratiques est relativement stable, entre 8,5 et 6,6 sorties par semaine.

Les graphiques ci-dessous montrent les effets de substitution entre les motifs de sorties selon les âges de la vie, et en fonction du genre. Pour tous, l'entrée dans les âges de la vieillesse est synonyme d'un regain des pratiques urbaines liées à la vie sociale, au détriment du travail pour les hommes et au détriment du travail et des contraintes domestiques pour les femmes.

Figure 1 - Mobilités par motif, selon l'âge et le sexe
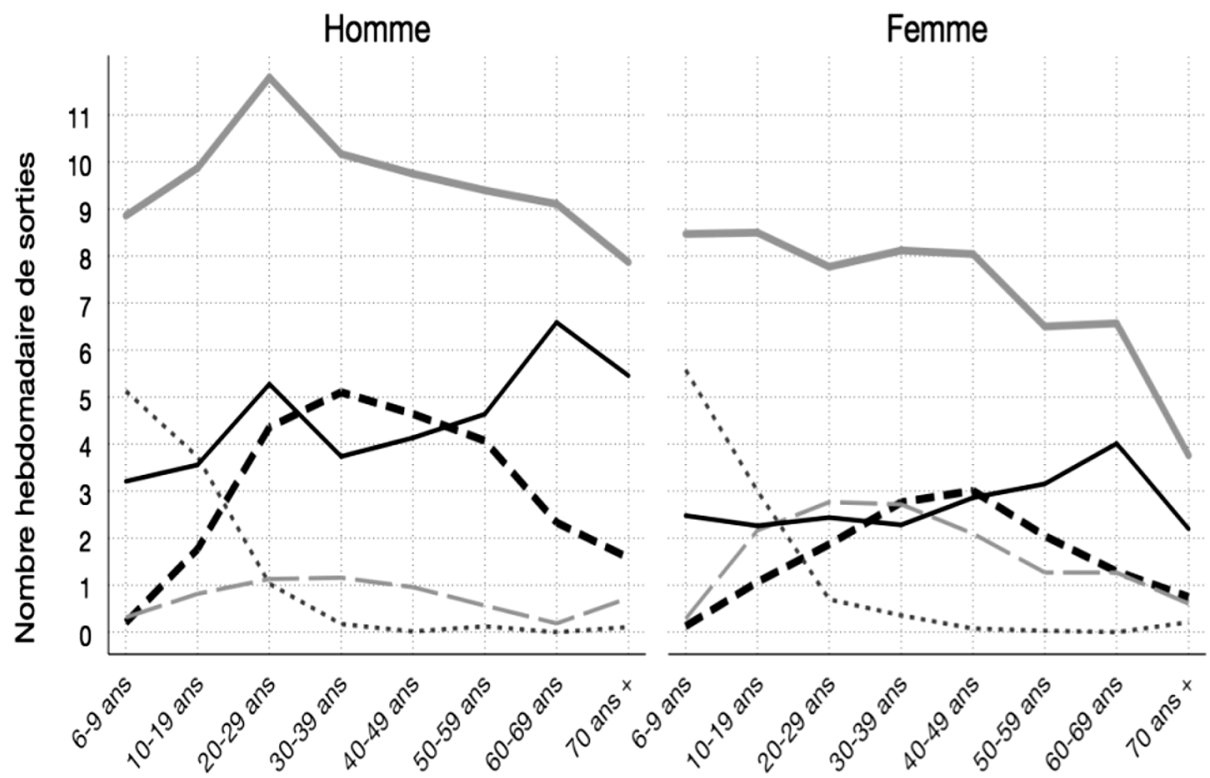

\title{
Âge des individus
}

\author{
- Tous les motifs - Pour le travail $\cdots$ les études - sphère domestique - vie sociale/autre \\ Source : Enquête « Ouga. 2009 »
}

L'appartenance à une catégorie socioprofessionnelle ne constitue pas non plus un facteur de différenciation fort, hormis pour les inactifs (jeunes et personnes âgées) et les travailleurs à domicile ; ces derniers s'épargnent en effet les contraintes liées au travail, qui ne sont pas compensées par d'autres motifs. Pour le travail, les catégories les plus élevées - salarié des secteurs public et privé, employeur ou patron - et les plus précaires - journalier, apprenti, indépendant avec ou sans local - ne se différencient pas, autour de 5,5 sorties hebdomadaires sont comptabilisées. 
Le lieu de résidence ne distingue pas non plus les individus, hormis s'ils résident dans les quartiers non lotis peu denses. Pour ces derniers, la part moindre des sorties associées à la vie domestique explique ce léger décrochage ; alors que pour les autres quartiers, elle est environ de deux sorties par semaine, elle n'est que d'une sortie pour les quartiers non lotis. La vie sociale à l'extérieur occupe également une place moindre chez les résidents des quartiers non lotis épars. Le réseau social des nouveaux occupants se trouve éloigné de leur logement, dans un environnement peu dense.

Ainsi, l'analyse des pratiques urbaines ne fait pas apparaître de fortes différenciations entre les individus. Ils sont donc en mesure de se déplacer pour leurs activités essentielles, indépendamment de l'équipement, de leur niveau de vie (absolument pas discriminant), de leur âge ou sexe. Ils gardent même la liberté d'arbitrer entre les fonctions et les contraintes de la vie urbaine.

Cependant, compte tenu des inégalités dans la hiérarchie modale, tous les individus n'ont pas la possibilité d'accéder aux mêmes lieux dans la ville, dans les mêmes conditions. Alors que les pratiques de certains habitants sont restreintes au quartier, d'autres ont la capacité - ou sont contraints - d'élargir leur espace de vie à toute la ville.

\section{Du quartier à la ville}

Les pratiques urbaines définissent l'espace de vie, la question de son étendue a été introduite à partir du domicile. En effet, parcourir toute la ville ou seulement son quartier n'a pas les mêmes implications sociales ou économiques, au vu de l'inégale concentration des ressources dans l'espace urbain (emplois, services, réseaux sociaux...). L'enquête permet d'apprécier les pratiques urbaines selon qu'elles se font «dans le quartier» ou « hors du quartier ${ }^{13}$.

Les sorties de proximité sont les plus nombreuses, d'une courte majorité, soit $58 \%$ dans le quartier. Mais c'est la pratique féminine qui fait la différence (64\% des déplacements proches) alors que pour les hommes la répartition est équilibrée. $\mathrm{Du}$ point de vue des individus, toutes sorties agrégées, la plus grande mobilité des hommes creuse l'écart. En effet, le nombre de sorties proches est pratiquement identique pour les deux sexes (5,8 pour les hommes, 5,6 pour les femmes). Mais les hommes sortent presque deux fois plus souvent du quartier ( 4,5 sorties pour 2,4 de la part des femmes). Ce qui implique des trajets plus longs (près du double du temps de déplacement), nécessitant plus impérativement une motorisation. Ces différences reflètent à la fois la plus grande activité professionnelle des hommes et la localisation plus lointaine et dispersée de leurs emplois.

13. Le « quartier» ne constitue pas à Ouagadougou une division administrative de l'espace ; il renvoie plutôt à un espace vécu et nommé par les habitants. La distinction entre les déplacements « dans le quartier» et « hors du quartier » a donc été laissée à l'appréciation des habitants. Les nombres moyens de sorties par individus ne sont ici calculés que sur les personnes mobiles pour un motif ou pour un autre. Les sédentaires « absolus » sont exclus de la population de référence puisqu'il s'agit de ne considérer que les sorties du quartier. 
La manière dont chacun va vers la ville au-delà de son quartier exprime aussi les différences socio-économiques de l'espace de vie. À considérer la variation de ce paramètre avec le niveau de vie du logement, on constate que c'est la part de la mobilité hors du quartier qui augmente avec l'aisance économique (du simple au double), jusqu'à dépasser les déplacements proches parmi les plus aisés, alors que parmi les plus pauvres, ils sont trois fois moins importants. Il s'agit bien de l'élément le plus discriminant de différenciation économique, alors que la mobilité totale ne varie pratiquement pas entre les plus pauvres et les plus aisés.

Figure 2 - nombre de sorties hebdomadaires dans et hors le quartier

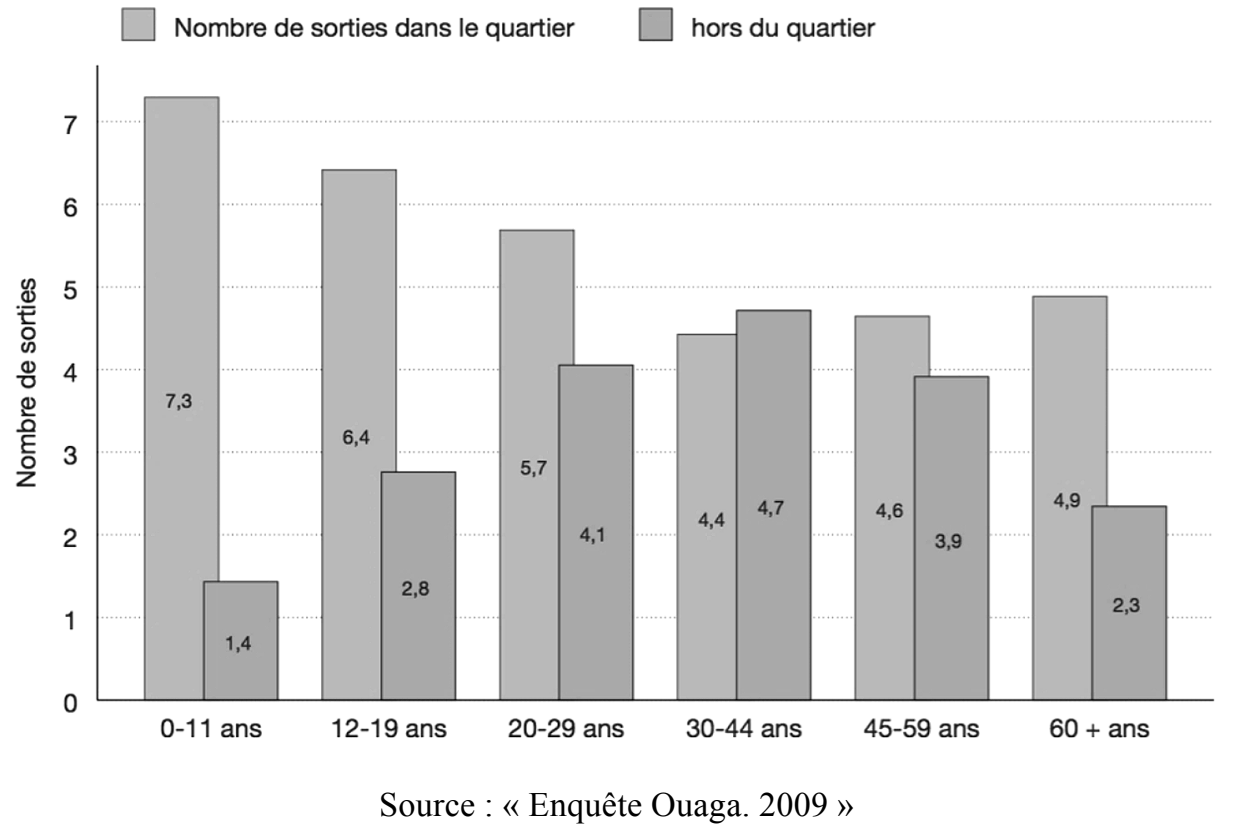

Le rapport à la ville change aussi au cours du cycle de vie, dans des proportions considérables, selon une distribution de l'externalité qui culmine à 30-49 ans, au milieu de la vie active. Les jeunes sortent peu de leur quartier (l'école, les amis sont proches) et même dans leur vingtaine, la mobilité proche prédomine, sans doute en raison de leur équipement plus faible. Les personnes plus âgées vivent également un repli sur le voisinage à raison de 2,3 sorties vers l'extérieur pour 4,9 dans le quartier (pour les 60 ans et plus), alors qu'entre 30 et 44 ans on fréquente plus la ville que son quartier $(4,7$ sorties pour 4,4$)$.

Intuitivement, la localisation dans la ville risque de conditionner la nécessité de sortir de son îlot qui peut manquer des aménités indispensables quand on s'éloigne du centre. D'autres facteurs interviennent comme l'équipement en moyens motorisés, le niveau de vie, l'âge moyen des habitants. Finalement, la différenciation est modérée. Dans le centre-ville d'avant 1980, des habitants en début et fin de cycle de vie bénéficiant d'une meilleure motorisation trouvent le 
meilleur équilibre entre les deux cercles de mobilité. Certes, l'externalité de la mobilité diminue vers la périphérie, mais modérément et de manière irrégulière : 3,7 sorties hors du quartier dans le centre, 3,3 dans le loti dense, 2,5 dans le loti épars. Se tourner vers la ville est imposé par le travail. Mais ce sont surtout les mobilités proches qui reculent dans le non loti ( 2 sorties en moins en grande périphérie par rapport au péricentre) ; un peu comme si le voisinage manquait d'attrait pour la vie sociale, la relative pauvreté réduisait la consommation, le temps manquait après les longs déplacements vers le travail.

Car finalement, se tourner vers la ville est d'abord une exigence du travail, de sa localisation. Ainsi le fonctionnariat exige trois fois plus de déplacements hors du quartier que de proximité (à un niveau très faible), le travail salarié privé et associatif, une fois et demie plus. Les journaliers trouvent à se loger près de leur lieu de travail et se déplacent très souvent dans le quartier ( 7 sorties hebdomadaires, un record avec les écoliers).

\section{Conclusion}

Le bilan des inégalités de mobilité à Ouagadougou est en demi-teinte. Capitale de taille intermédiaire à l'échelle régionale, Ouagadougou est une ville où les pratiques de mobilité ne permettent pas d'établir des différenciations importantes entre les individus. Les femmes ne sont pas forcément plus sédentaires que les hommes, seules les personnes âgées, en fin de vie active, se distinguent par leur sédentarité. Finalement, les pratiques de mobilité résultent d'un arbitrage entre mobilité contrainte ou non et sédentarité, elle aussi subie ou non.

Pourtant, si les pratiques ne révèlent pas de profondes inégalités, des contrastes apparaissent en fonction de la localisation dans la ville et de la possibilité d'utiliser ou non un moyen de transport. De nombreux Ouagalais sont soit dépourvus de véhicules individuels, soit disposent d'un véhicule de mauvaise qualité ; cette lacune se cumule souvent avec un logement dans des quartiers périphériques où les emplois et les services sont rares. Elle peut conduire à des situations d'immobilité contrainte ou de pénibilité dans les déplacements pour ceux qui n'ont accès qu'à la marche ou à un vélo et qui cumulent déficience du moyen de transport et localisation résidentielle périphérique. À l'inverse, ceux qui jouissent d'une localisation urbaine centrale peuvent accéder aux services et infrastructures de base, sans recourir à un moyen de transport.

Autant d'éléments qui renforcent le constat, déjà fait par de nombreux auteurs [Godard, 2002 ; Cima+ International, 2000 ; Bamas, 2003 ; SITRASS, 2003 ; Trans-Africa international, 2009 ; etc.], d'une « spécificité ouagalaise » dans le contexte régional : l'absence d'un système efficace de transports collectifs formels (les bus) ou artisanaux (les taxis collectifs) marginalise de fait tous ceux qui n'ont pas accès à un véhicule individuel. Le vélo, qui occupe à Ouagadougou une place inégalée dans les autres capitales africaines, permet de compenser cette absence pour ceux qui en disposent, notamment en périphérie non lotie où l'équipement 
en vélos est assez élevé et permet d'assurer des déplacements relativement rapides, nonobstant la vulnérabilité de ce mode de transport aux intempéries (chaleur ou pluie) et sa dangerosité. Cette situation interroge directement les politiques publiques, qui jusque-là ont réussi à dissuader la prolifération des transports collectifs artisanaux, mais sans réussir à mettre en place, en contrepartie, un système efficient de bus [Gouëset, 2010] ; cependant, mettre en place un système de transport en commun se heurte aussi à la capacité des usagers d'en assumer le coût. Or, les pratiques de mobilité sont partagées à Ouagadougou en fonction d'arbitrages individuels ou familiaux, qui permettent à pratiquement tout le monde de se déplacer. Un système de transport en commun efficient aurait-il la capacité non seulement à améliorer les possibilités de déplacement, mais aussi à maintenir les pratiques de mobilité à un niveau relativement élevé ?

Les pouvoirs publics devront sans doute s'attacher dans l'avenir au problème des transports collectifs, formels ou informels, car leur faiblesse n'est que partiellement compensée par les deux-roues et le vélo, qui constituent une alternative intéressante et rarement soutenue dans les métropoles africaines. Les perspectives démographiques plaident dans le même sens. Ouagadougou s'étend à un rythme élevé : au rythme actuel, la ville atteindra quatre millions d'habitants à l'horizon 2025, c'est-à-dire le double qu'en 2009. Le modèle d'étalement urbain ouagalais, avec des densités résidentielles très basses et le rejet de jeunes ménages en grande périphérie ne fait qu'amplifier le problème. La situation est préoccupante notamment pour les femmes, dont l'activité devient identique à celle des hommes une fois passé le cycle reproductif, mais qui ont difficilement accès à un véhicule individuel ; elle l'est plus encore pour les jeunes adultes, mieux éduqués que leurs aînés, mais qui doivent accompagner l'étalement de la ville sans toujours avoir les ressources suffisantes pour acquérir un moyen de transport motorisé. Cette situation n'est pas seulement injuste, elle est aussi contre-productive, puisque ces jeunes adultes représentent l'avenir économique de la capitale burkinabè.

\section{Bibliographie}

$\mathrm{B}_{\mathrm{ACQUE}}$ M.-H., $\mathrm{F}_{\mathrm{OL}} \mathrm{S}$. [2007], « L'inégalité face à la mobilité : du constat à l'injonction », Revue suisse de sociologie, vol. $33, \mathrm{n}^{\circ} 1$, p. 89-104.

$\mathrm{B}_{\mathrm{AMAS}} \mathrm{S}$. [2003], "Les transports urbains à Ouagadougou : diagnostic et perspectives », in CRET, Études urbaines à Ouagadougou, Burkina Faso, Pessac, Presses universitaires de Bordeaux, p. 59-90.

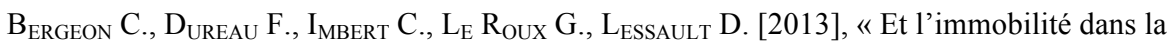
circulation? », e-migrinter, $\mathrm{n}^{\circ}$ 11, p. 3-6 : https://e-migrinter.revues.org (consulté le 18 août 2017).

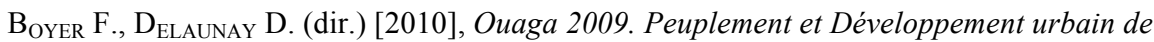
Ouagadougou, Ouagadougou, Institut de recherches pour le développement (IRD), Service de coopération et d'action culturelle (SCAC).

$\mathrm{C}_{\mathrm{IMA}}+\mathrm{I}_{\mathrm{NTERNATIONAL}}$ [2000], Étude d'amélioration des conditions de déplacements dans l'agglomération de Ouagadougou, ministère des Transports et du Tourisme, Direction des études et de la planification, Ouagadougou, 3 volumes, rapport de synthèse et annexes. 
C $_{\text {USSET }}$ J.-M. [1997], « Mobilité des deux roues et politique de transport à Ouagadougou et à Hanoi », in $\mathrm{F}_{\text {IGUEROA }} \mathrm{O}$., $\mathrm{G}_{\mathrm{ODARD}} \mathrm{X} ., \mathrm{H}_{\mathrm{ENRY}} \mathrm{E}$. (dir.), Mobilité et politiques de transport dans les villes en développement, Actes des journées spécialisées $\mathrm{I}_{\text {NRETS }}, 30-31$ janvier 1997, Actes n ${ }^{0} 55$, p. 87-104.

D Elaunay $_{\text {D., F }}$ OuRnier J.-M. [2014], « Mesurer le capital de mobilité pour évaluer les différenciations sociodémographiques et intra-urbaines de l'accessibilité. Le cas de la zone métropolitaine de Santiago du Chili », Revue Tiers Monde, nº 2, p. 131-149.

$\mathrm{D}_{\text {IAZ }} \mathrm{O}_{\text {LVERA }}$ L., $\mathrm{P}_{\text {LAT }}$ D. [1996], « Les mobilités quotidiennes à Ouagadougou », Cahiers des sciences humaines, vol. 2, $\mathrm{n}^{\circ}$ 32, p. 293-309.

$\mathrm{D}_{\text {IAZ }} \mathrm{O}_{\text {LVERA }}$ L., $\mathrm{P}_{\text {LAT }}$ D. [1997], " Confisquée, partagée, consensuelle. La voiture à Ouagadougou », in $\mathrm{F}_{\mathrm{IGUEROA}} \mathrm{O}$., $\mathrm{G}_{\mathrm{ODARD}} \mathrm{X}$., $\mathrm{H}_{\mathrm{ENRY}} \mathrm{E}$. (dir.), Mobilité et politiques de transport dans les villes en développement, INRETS, Actes $\mathrm{n}^{\circ}$ 55, p. 213-225.

$\mathrm{D}_{\text {IAZ }} \mathrm{O}_{\text {LVERA }}$ L., $\mathrm{P}_{\text {LAT }}$ D., $\mathrm{P}_{\text {OCHET }}$ P. [1998], Villes africaines au quotidien, Lyon, Laboratoire d'économie des transports (LET).

$\mathrm{D}_{\text {Iaz }} \mathrm{O}_{\text {lvera }}$ L., $\mathrm{P}_{\text {Lat }}$ D., $\mathrm{P}_{\text {Ochet }}$ P., $\mathrm{S}_{\text {Ahabana }}$ M. [2010], « Entre contraintes et innovation : évolutions de la mobilité quotidienne dans les villes d'Afrique subsaharienne ", Espace populations sociétés, $\mathrm{n}^{\mathrm{o}} 2-3$, p. 337-348.

$\mathrm{D}_{\text {UREAU }}$ F., G GuëSET V. [2010], « Formes de peuplement et inégalités de déplacements. L'évolution des mobilités quotidiennes dans deux périphéries populaires de Bogotá : Soacha et Madrid (1993-2009) », Revue Tiers Monde, n 201, p. 131-156.

$\mathrm{F}_{\mathrm{OL}} \mathrm{S}$. [2009], La mobilité des pauvres. Pratiques d'habitants et politiques publiques, Paris, Belin.

$\mathrm{F}_{\mathrm{OL}} \mathrm{S}$. [2010], « Encouragement ou injonction à la mobilité ? », Projet, $\mathrm{n}^{\mathrm{o}} 314$, p. 52-58.

$\mathrm{G}_{\mathrm{ODARD}} \mathrm{X}$. [2002], Les transports et la ville en Afrique au sud du Sahara. Le temps de la débrouille et $d u$ désordre inventif, Paris, Karthala, INRETS.

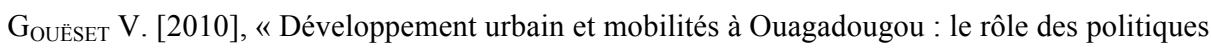
publiques », in $\mathrm{B}_{\text {OYer }}$ F., $\mathrm{D}_{\text {Elaunay }}$ D. (dir.), " Ouaga 2009 », Peuplement et développement urbain de Ouagadougou, Ouagadougou, Institut de recherches pour le développement (IRD), Service de coopération et d'action culturelle (SCAC), p. 136-167.

$\mathrm{K}_{\mathrm{ORSU}}$ E., W $\mathrm{W}_{\text {ENGLENSKI }} \mathrm{S}$. [2010], « Job accessibility, residential segregation and risk of longterm unemployment in the Paris Region », Urban studies, vol. 47, $\mathrm{n}^{\circ} 11$, p. 2279-2324.

$\mathrm{L}_{\mathrm{ANNOY}} \mathrm{P}$., $\mathrm{R}_{\mathrm{AMADIER}} \mathrm{T}$. (dir) [2007], La mobilité généralisée. Formes et valeurs de la mobilité quotidienne, Louvain-la-Neuve, Academia-Bruylant.

$\mathrm{L}_{\mathrm{E}} \mathrm{B}_{\mathrm{RETON}}$ E. [2005], Bouger pour s'en sortir. Mobilité quotidienne et intégration sociale, Paris, Armand Colin.

$\mathrm{M}_{\mathrm{IGNOT}}$ D. [2004], « Transport et justice sociale », Reflets et perspectives de la vie économique, vol. $43, n^{\circ} 4$, p. 123-131.

$\mathrm{P}_{\mathrm{RAT}}$ A. [1996], « Ouagadougou, capitale sahélienne : croissance urbaine et enjeu foncier », Mappemonde, vol. 1, n 96, p. 18-24.

$\mathrm{S}_{\text {ITRASS }}$ [2003], Étude complémentaire pour l'évaluation d'un programme de transports urbains à Ouagadougou. Rapport final, Ouagadougou, ministère des Infrastructures, des Transports et de l'Habitat, projet sectoriel des transports.

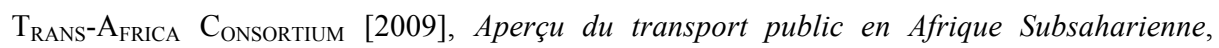
Bruxelles, Union internationale des transports publics. 